\title{
CONTRIBUCIONES AL ANÁLISIS GEOGRÁAICO Y DIAGNÓSTICO TERRITORIAL DE CASTILLA Y LEÓN
}

\author{
Lorenzo LÓPEZ TRIGAL \\ Departamento de Geografía y Geología, Universidad de León
}

Recibido: 01/06/2010

Aceptado: 07/06/2010

RESUMEN: El objetivo ha sido llevar a cabo una revisión analítica en tres direcciones que nos aproximen al conocimiento geográfico de Castilla y León, una primera, relativa a la contribución esencialmente de geógrafos al análisis de la región castellano-leonesa durante el último medio siglo; una segunda, en cuanto a la disección de esquemas de referencia territorial en las distintas escalas, habituales en su mayor parte en la explicación geográfica; y una tercera, relativa a la presentación de ciertos rasgos de la situación y proyección socioeconómica y estratégica de la región, apoyándonos en la ya abundante bibliografía y la propia investigación regional.

$P A L A B R A S C L A V E$ : análisis geográfico, diagnóstico territorial, proyección estratégica, región, Castilla y León.

CONTRIBUTIONS TO GEOGRAPHIC ANALYSIS AND TERRITORIAL DIAGNOSTIC OF CASTILE AND LEON

ABSTRACT: The objective was to carry out a three-fold analytic review intended to enhance geographical awareness of the Castile and Leon region of Spain. The first strand corresponded essentially to the contribution of geographers to the study of Castile and Leon over the last half century. The second had to do with the investigation of schemes for territorial reference on different scales, for the most part customary in geographical explanations. The third related to the presentation of certain features of the socio-economic and strategic projection of the region, on the basis of an already abundant bibliography and regional research undertaken.

KEY WORDS: geographical analysis, territorial diagnosis, strategic projection, region, Castile and Leon.

\section{CONTRIBUTIONS AU ANALYSE GEOGRAPHIQUE ET DIAGNOSTIC TERRITORIAL DE CASTILLE-ET-LEON}

RÉSUMÉ: La révision de trois grands axes analytiques nous permet de renforcer notre connaissance géographique de la région de Castille et León. Le premier axe étudie la contribution, au cours des 50 dernières années, des géographes dans l'analyse de la région de Castille et León. Le second consiste en une analyse détaillée des schémas de référence territoriale à différentes échelles, un concept clé de l'explication géographique. Le troisième, qui s'appuie sur l'abondante littérature et sur la recherche régionale, a pour objectif de dégager les principales caractéristiques de la situation et d'élaborer une planification socio-économique et stratégique de la région. 
MOTS-CLÉS: analyse géographique, diagnostic territorial, planification stratégique, région, Castille-et- León.

\section{CONTRIBUIÇÕES AO ANÁLISE GEOGRÁFICO E DIAGNÓSTICO TERRITORIAL DE CASTELA E LEÃO}

RESUMO: O objetivo foi realizar uma revisão analítica em tres direções que nos aproximem ao conhecimento geográfico de Castela e Leão, a primeira, refere-se à contribuição principalmente dos geógrafos ao análise da região castelano-leonesa durante o último meio século; a segunda, quanto à divisão de esquemas de referência territorial nas diferentes escalas, que se encontram na sua maior parte na explicação geográfica; e uma terceira, relativa à presentação de determinados traços da situação e projeção estratégica e socioeconómica da região, usando a ja abundante bibliografia e a investigação regional própia.

PALAVRAS-CHAVES: análise geográfico, diagnóstico territorial, projeção estratégica, região, Castela e Leão.

\section{LAS APORTACIONES GEOGRÁFICAS SOBRE LA REGIÓN}

Desde diferentes visiones y universidades españolas los geógrafos han venido contribuyendo al estudio regional y subregional de Castilla y León, después de contar como precedentes relevantes el estudio de la Meseta septentrional llevado a cabo en la primera monografía regional de la incipiente escuela geográfica española (TERÁN, 1958), a la que siguen más tarde capítulos de sendas obras regionales de España de los catedráticos de los departamentos de Geografía de Valladolid y Salamanca (GARCÍA FERNÁNDEZ, 1968; CABO ALONSO, 1975). Más tempranos son los estudios pioneros en la aproximación subregional, comenzando por los de ámbito provincial iniciados en León (LÓPEZ TRIGAL, 1980) por Modesto Medina Bravo, inspector de enseñanza primaria en una fecha muy temprana, 1927, y posteriormente una primera generación de tesis doctorales, destacando las de José Luis MARTín GALINDO, 1948, sobre la Maragatería, autor bien significativo y en buena medida al margen de la Academia universitaria, junto a José Manuel RUBIO RECIO, 1955, sobre la Ribera del Órbigo, o Ángel CABO ALONSO, 1960, sobre la estructura agraria de la provincia de Salamanca. A la vez otros análisis irán sumándose a los primeros frutos de la investigación geográfica, centrada por entonces, de modo general, en estudios de referencia y escala local, comarcal y subregional, entre los que destacar trabajos agraristas así como el dedicado al crecimiento y estructura urbana de Valladolid, 1974, de Jesús GARCÍA FERNÁNDEZ.

Asimismo, se pueden considerar precedentes de cierta relevancia en el ámbito del análisis sectorial ciertos ensayos imbuidos del regionalismo renaciente como el Informe económico sectorial del SERVICIO DE ESTUDIOS DEL BANCO DE BILBAO PARA CASTILLA Y LEÓN (1971), los estudios de reconocimiento territo- 
rial debidos a la consultora EYSER (1981), además de algún texto sociológico centrado en las tierras castellanas, en el éxodo de sus habitantes y las dificultades de las explotaciones agrarias (SOREL, 1975) o un ensayo regionalista y del momento coyuntural (CARRASCO, 1978). En un contexto académico fue convocado por Eduardo Martínez de Pisón, por entonces profesor en la Universidad Complutense, la "Primera Semana sobre Geografía de Castilla" en la ciudad de Segovia, 1977, y dedicada al medio natural, el espacio rural y las ciudades y rematada con una jornada sobre el "problema regional en Castilla" en relación con propuestas de regionalización, problemas metodológicos para una regionalización y perspectivas del desarrollo regional. Este primer encuentro de los geógrafos conecta con el ideario de la Transición política que reiniciará el proceso de regionalización en España, surgiendo también por entonces grupos de acción como el 'Instituto Regional Castellano-Leonés', que aúna a profesionales y universitarios con la idea de "recuperar la tradición histórica, ahondar en la identidad territorial y engancharse al proceso regionalizador y el desarrollo económico". Aunque al mismo tiempo, en el debate regional, entran grupos provincialistas y comarcalistas alimentados por la diversidad de los territorios, el recelo y la lucha por la capitalidad de las nuevas instituciones y que aún persisten, treinta años después, con cierta representatividad en el caso de León.

Durante esa misma década se sucederá una segunda generación de tesis doctorales, sobre distintos ámbitos geográficos de la región castellano-leonesa, en las que en unas se sigue el rumbo marcado por los estudios anteriores, aunque con una mayor especificidad y detenimiento en el tratamiento: Pedro PLANS SANZ DE BREMOND ("La Tierra de Campos"), Gonzalo BARRIENTOS ("El Valle Alto del Tormes"), Fernando Molinero ("La Tierra de Roa: la crisis de una comarca vitícola tradicional"), Juan José SANZ DONAIRE ("El corredor de Béjar"), Valentín CABERO DIÉGUEZ ("Estudio geográfico de un espacio marginal en las montañas galaico-leonesas: la Cabrera"). Mientras que en otras se participa de nuevos temas y/o enfoques de tipo teórico-cuantitativo: Eduardo MARTÍNEZ DE PISÓN ("Segovia. Evolución de un paisaje urbano"), José ORTEGA VALCÁRCEL ("La transformación de un espacio rural: las Montañas de Burgos"), Lorenzo LÓPEZ TRIGAL (“La Red Urbana de León") o el alemán Eibken LIEBHOLD ("Las estructuras de lugares centrales y de región funcional en los esquemas de poblamiento de la Meseta norte en España", publicada en Bonner Geographische Abhaudlungen, $\mathrm{n}^{\mathrm{o}} 60,1979$ ).

Posteriormente, a comienzos de los años 1980, se iniciará propiamente en los estudios geográficos, al igual que en los económicos, sociológicos, antropológicos e históricos, la normalidad en el análisis regional, coincidiendo con la consolidación de la regionalización autonómica. Trayectoria que aparece encabezada por la publicación de un ensayo de la situación involutiva y las "manifesta- 
ciones aparentes del desarrollo" de la región, seguido de una reflexión sobre la reforma agraria y la ordenación industrial, por parte de Jesús GARCía FERNÁNDEZ (1981) -FIGURA 1-, quien en años anteriores había elaborado igualmente certeros estudios sobre la España Atlántica en ediciones de amplia difusión nacional, que se convertirán por entonces en libros cabecera de universitarios, técnicos y políticos que se precien de esta "región demasiado heteróclita" según sus palabras. El mismo va a realizar una atinada y minuciosa reflexión sobre la percepción del espacio de Castilla y la tradición historicista en una obra posterior, que sitúa una vez más la cuestión regional en distintos frentes, mostrando su propia opinión, más bien ecléctica, al respecto del debate regionalizador: "tampoco consideramos un inconveniente que la región política no coincida con lo que es la concepción geográfica de un conjunto de regiones... Y es bajo este doble aspecto - región política y regiones geográficas - como la Geografía puede ser útil, dando a conocer la realidad de su espacio" (GARCíA FERNÁNDEZ, 1985, 312).

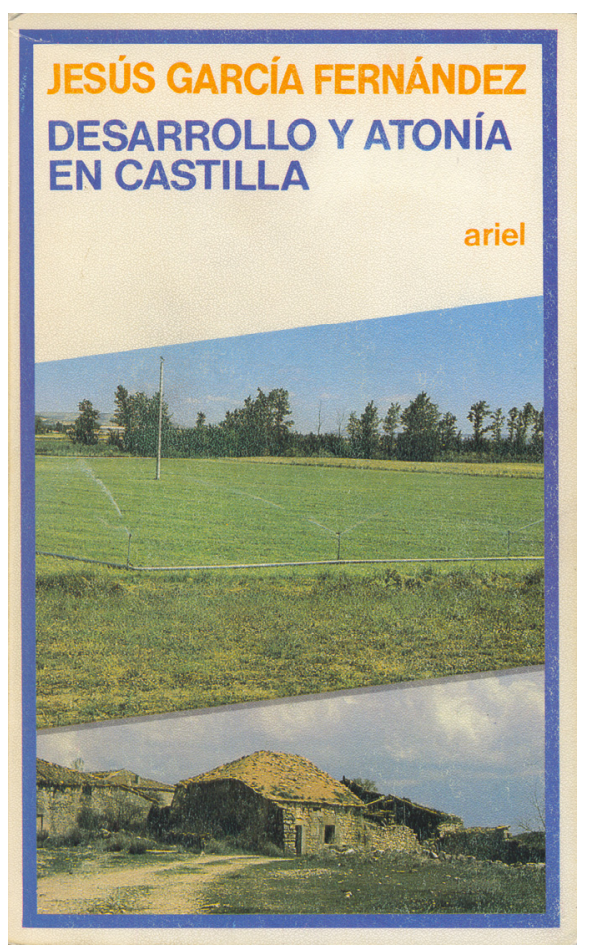

Fig. 1. Portada del libro de J. García Fernández, Desarrollo y atonía en Castilla.
En el contexto de la convocatoria del I Congreso de Cultura castellano-leonesa promovido por el recién creado Consejo General de Castilla y León se aspira a asentar unas bases históricogeográficas en un "marco adecuado para que de este conocimiento nacerá el amor hacia todo lo nuestro y nuestra cultura", siendo la temática correspondiente a la geografia coordinada por García Fernández -Geografía: Castilla La Vieja-León como región geográfica. La realidad de nuestras comarcas- (AAVV, 1982) - FigurA 2-. A este fin tiene lugar un primer encuentro celebrado en Burgos que recibe una nutrida representación de universitarios y docentes, si bien sólo un tercio de los textos presentados adoptan un tratamiento de escala regional, y distribuidos en tres bloques (los espacios eco-

lógico, rural e industrial-urbano), resultado en su mayor parte de "pacientes y 
cabales investigaciones" como aprecia el mismo García Fernández. En esta misma clave regionalista se contribuye con la colaboración de geógrafos al estudio y propuesta de comarcalización (EYSER, 1984-1985), al análisis del medio físico, delimitación de unidades y estructura territorial por provincias (AAVV, 1988), y a la elaboración de una guía escolar de síntesis para la docencia en ciencias sociales y humanas (AAVV, 1987), todo ello al amparo de la Junta de Castilla y León.

Concluida la transición regionalizadora, el respaldo definitivo a los estudios regionales se sostendrá en buena parte por iniciativa de editorial Ámbito, de nueva aparición con sede en Valladolid e impulsada por un grupo de profesores a cuyo frente estará el historiador Julio Valdeón, con un claro signo regionalista y buscando una difusión más allá del entorno universitario. Se va a editar, en primer lugar, en formato de libros de bolsillo, una serie que encabeza en su segunda entrega Valentín CABERO (1982) -FIGURA 3- en un ajustado tratamiento del espacio geográfico castellano-leonés con el que se inician de hecho las bases de la geografía de Castilla y León, a partir de sus condiciones ecológicas y

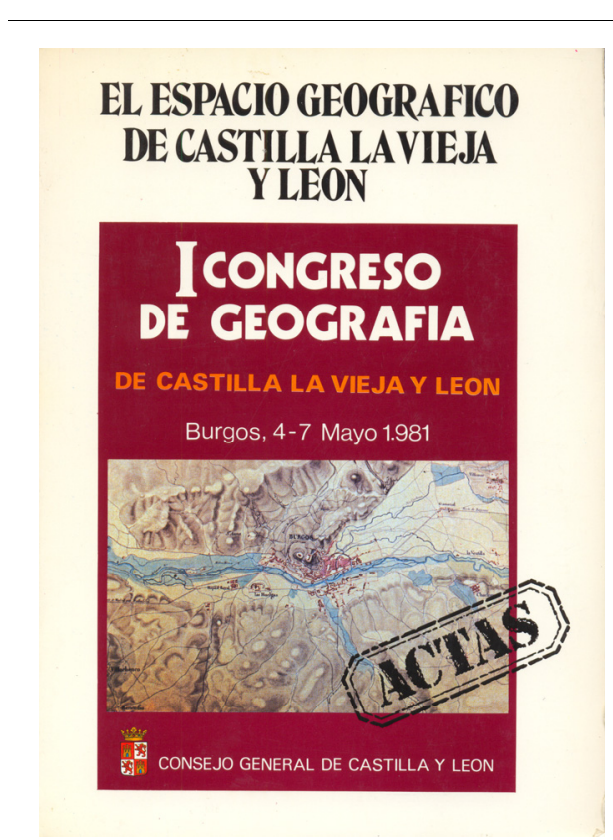

Fig. 2. Portada de El espacio geográfico de Castilla la Vieja y León. recursos naturales, para seguir con trabajos más específicos dedicados a la agricultura, la industria y el transporte por parte de geógrafos de la "escuela de Valladolid" (MOLINERO, 1982, MANERO, 1983 y PASTOR, 1986).

En segundo lugar, la editorial presentará un proyecto de larga discusión, que se plasma en los volúmenes correspondientes de la historia, historia del arte y geografía regional de Castilla y León (de la que queda descolgado García Fernández por propia voluntad), con un tratamiento ambicioso e innovador en lo temático y gráfico (CABO Y MANERO, dirs. 1987-1992) -FIGURA 4-, que "consiguió lo que podría adivinarse entonces como el arranque (de una geogra-fía) regionalista sólida... en clave y perspectiva de regionalismo en cierto modo militante" (CARASA, 2003, 569). Se orienta la obra a "redescubrir la región", esto 
es, el esclarecimiento de los factores que conforman el territorio mediante la elaboración de un diagnóstico de la región y sus diferentes comarcas (espacios tradicionales y espacios renovados), como eje vertebrador del análisis geográfico regional de los espacios naturales, la actividad agraria, la industria y recursos, la población, la transformación urbana, y la articulación del territorio, que se completará con una síntesis y planteamientos de futuro, a modo de "resumen no técnico" de cara a adoptar políticas de actuación eficaces y sintonizadas con la realidad del territorio.

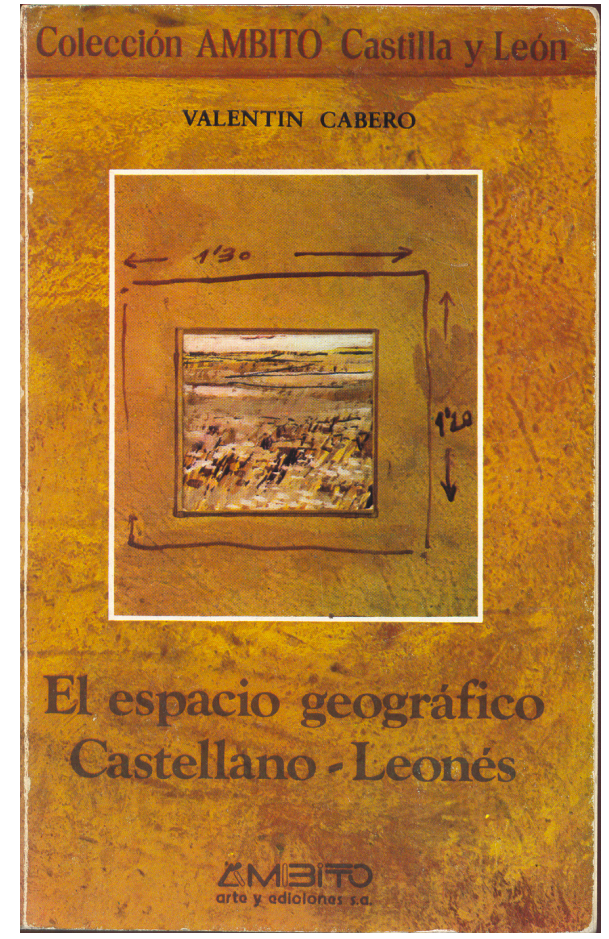

Fig. 3. Portada del librode Valentín Cabero, El espacio geográfico castellano-leonés.
En los años inmediatos comienza a impartirse en los planes de la Licenciatura de Geografía en España la Geografía de la propia región, lo que, unido a las investigaciones realizadas a la par en esta misma línea regional y la demanda de ediciones de difusión general, anima a que se desarrollen diferentes proyectos editoriales de ámbito nacional (Manero, 1987, López Trigal, 1987 y 1994; Cabo y Manero, 1990).

Posteriormente no se han editado nuevas monografías de geografía regional, sino más bien proyectos cartográficos y bibliográficos de alcance muy diverso, promovidos a menudo desde instituciones regionales, caso de estudios pluridisciplinares sobre la economía y el territorio de la región en ciertos informes y monografías, destacando en esta línea la Consejería de Economía y Hacienda de la Junta de Castilla y León con la convocatoria anual de Congresos Regionales y a través de la colección "Estudios Económicos" (ejemplo significativo el volumen AAVV, 1998), el Consejo Económico y Social de Castilla y León, con aportación de múltiples Informes, entre otros los dedicados a la población como recurso y la ordenación territorial (CES, 1998 y 2003), la actividad de la Asociación Castellano-Leonesa de Ciencia Regional, con reuniones de estudios dedicados a analizar la integración en Europa y a los sectores económicos, la edi- 
ción de la revista institucional Medio Ambiente desde 1994 a 2003, con atención a los diagnósticos regionales y comarcales y las metodologías de aplicación de análisis regional, o la Revista de Economía y Finanzas de la entidad Caja Duero, caso del monográfico dedicado a la región (2004). Asimismo, al igual que en otras Comunidades Autónomas, ha sido relevante, a efectos de aportación al estudio regional, la redacción y debate de los documentos de Directrices de Ordenación Territorial (TALLER DE IDEAS, 1996, 2000).

Se constata así que ha habido continuidad de los estudios de escala regional desde las tesis doctorales y los diversos análisis específicos sobre temáticas de población, las actividades económicas, el medio físico, las ciudades, o la ordenación territorial de Castilla y León... publicados en revistas geográficas nacionales y regionales, en contribuciones con ocasión de reuniones científicas de la Asociación de Geógrafos Españoles y a través de proyectos de investigación. A la vez, en otras escalas, han continuado realizándose estudios de sintesis subregional, provincial y comarcal de desigual entidad, difundidas a través de fascículos semanales en periódicos, artículos de revistas provinciales,

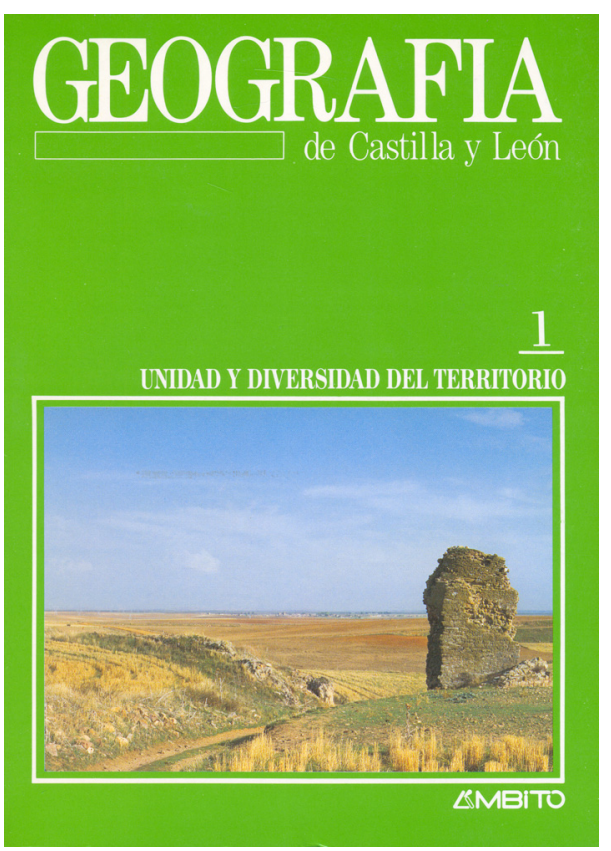

Fig. 4. Portada libro de Geografía de Castilla y León, de Ámbito . cuadernos y volúmenes de editoriales universitarias e institucionales. En particular, la revista Polígonos es, si acaso, una fiel muestra de la labor desarrollada en este campo a través de los 18 volúmenes que van desde su aparición en 1991, conteniendo análisis a escala de la región castellano-leonesa o de la cuenca del Duero y desde una temática diversa en una decena de artículos.

En general, las publicaciones, que sobre esta región o sus distintos ámbitos espaciales han ido apareciendo en las tres últimas décadas, presentan una marcada visión regional (e incluso "regionalista") y crítica (denunciando problemas y enunciando alternativas al modelo territorial y socioeconómico) del territorio y la sociedad de referencia, al igual que ha sido el caso del resto de geógrafos 
españoles en múltiples análisis espaciales de las diferentes Comunidades Autónomas. Más bien parece que los geógrafos españoles, a partir de 1980, hemos aceptado como "espacio de trabajo" el de las propias regiones autonómicas, como ha sido el caso evidente de Castilla y León.

\section{GEOIDEAS PARA UNA REORGANIZACIÓN TERRITORIAL}

A la hora de estudiar el sistema u organización territorial de cualquier región debemos situarnos en los diferentes ámbitos espaciales y escalas que se posicionan como marco de referencia, esto es, espacios que encajan en diferente grado unos en otros, a semejanza de las figuras superpuestas de una muñeca rusa, y que pueden servirnos de geoideas para el análisis regional y subregional, estando el proceso abierto a diferentes escalas macrorregional, regional y subregional, que incluye a su vez, no olvidemos, la unidad provincial. Entendemos que es siempre necesario el manejo de diferentes escalas, y máxime a la hora de analizar e intervenir en territorio tan extenso e interconectado en todas sus direcciones con otros territorios como es Castilla y León.

A) Pues bien, desde una escala macrorregional se perfilan territorios diferenciados e ideados hasta ahora más bien por los expertos y estudiosos. En primer término, el Suroeste de Europa, ámbito casi coincidente con los Estados ibéricos y sistemas urbanos nacionales español y portugués, que disponen de una interrelación cada vez más articulada, entre los cuales se interpone la línea fronteriza internacional a modo de interfaz, con sus respectivos archipiélagos insulares, más Andorra, Gibraltar, Ceuta y Melilla, y que en aplicación del modelo de la centralidad agrupa los territorios, ciudades y regiones de distinta dimensión y funcionalidad, conectando las redes de ciudades españolas con franjas del Sur de Francia y del Norte de Marruecos.

En segundo término, el correspondiente al troceado del territorio ibérico en clusters, tipo cuadrantes territoriales, siguiendo un criterio de traza geométrica y regional en el marco del sistema urbano y territorial, tal como se muestra-FIGURA 5-, sin olvidar ciertos elementos en presencia como son las relaciones más densas entre España y Portugal, la delimitación administrativa de las Comunidades Autónomas españolas y en menor medida de las Regiones de Coordinación portuguesas, la selección de corredores europeos de comunicación además de la propia dinámica de las redes o sistemas urbanos. Así, resulta, por ejemplo, que podemos apreciar, dentro del denominado "tercio Norte peninsular", diferentes cuadrantes: en primer término, el Cuadrante Centro-Norte español, formado por la región de Castilla y León más las regiones cántabra y asturiana, y en segundo término, el que engloba el occidente de la región castellano-leonesa (provincias de León, Zamora y Salamanca) en el Cuadrante Noroeste, que incorpora además regiones de España (Galicia y Asturias en el pri- 
mer supuesto o Galicia, Asturias y Cantabria en el segundo) y de Portugal (Región Norte). En ambos casos, conjuntos de territorios suprarregionales de una gran segmentación o compartimentación entre los distintos subsistemas territoriales, con desiguales interrelaciones y de fracturas de fronteras al lado de bordes montañosos.

Figura 5. Cuadrantes territoriales del sistema urbano ibérico

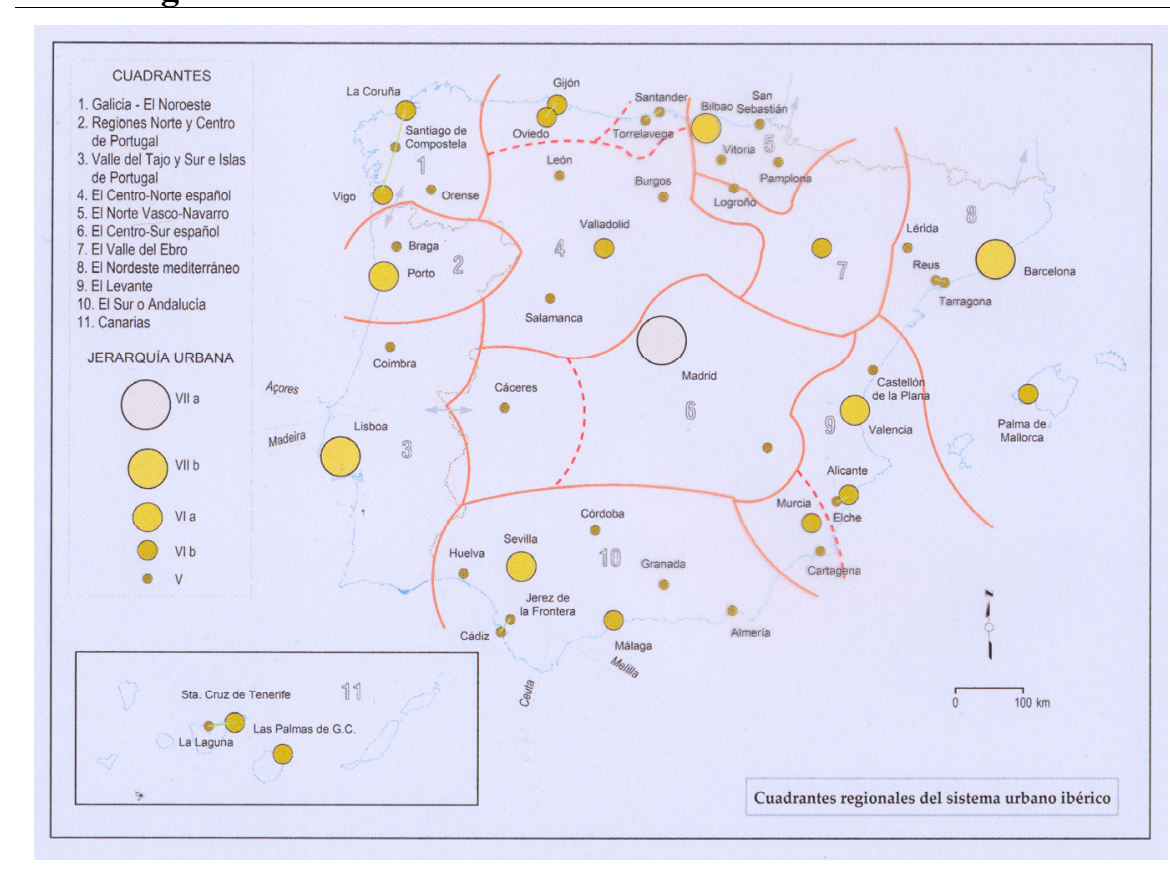

En tercer término, se viene manejando la disposición de dos grandes espacios macrorregionales de la geografía europea, de un lado, la Fachada Atlántica, en su tramo ibérico, que va desde el País Vasco hasta Andalucía Occidental, con la que conecta en muchos aspectos la región de Castilla y León, y de otro lado, las Regiones Interiores, en las que prima la centralidad de Madrid. En ambos casos se trata de denominaciones que se han ido consolidando en el argot comunitario de la Europa de las Regiones.

En cuarto término, la Eurorregión del Duero, ejemplo de región natural, superpuesto al ámbito de la Cuenca fluvial del Duero, compartida por España y Portugal, a modo de región-proyecto o espacio para el desarrollo común en el marco de la Unión Europea entre Castilla y León y la Región Norte de Portugal, espacio que encaja particularmente en buena parte de la región y que tiene co- 
mo instrumento privilegiado la cooperación transfronteriza, y por ello ya asumido recientemente por los gestores políticos.

B) Prosiguiendo en este análisis, a escala regional, corresponde el propio ámbito de la Comunidad Autónoma de Castilla y León, que "comprende el de los municipios integrados en las provincias..." (artículo 2, Estatuto de Autonomía, 1983) como entidad territorial básica de la región. En la reforma de Estatuto de 2007, la región es definida en su Preámbulo desde un enfoque historicista como "comunidad histórica y cultural" compuesta por los antiguos Reinos de Castilla y de León. En cualquier caso, se trata de una región también geográfica, como advierten, como hemos observado anteriormente, los estudiosos de la geografía española. Una gran región en extensión y conformada por subregiones y espacios de borde que tejen un territorio no tan homogéneo como a simple vista pareciera la inmensidad de su estepa.

C) En una escala subregional se presentan múltiples posibilidades en el diseño de espacios. Así, en primer lugar, los correspondientes a las áreas de atracción de las cuatro mayores ciudades, esto es, la delimitación de las subregiones de León, Salamanca, Burgos y Valladolid, animadas por flujos dinámicos bidireccionales de cada una de estas ciudades y su área, superpuesta a la esfera provincial, como es el caso, debido a la atracción de ciudades capitales subregionales de mayor rango, de la provincia de Zamora, repartida de hecho en dos zonas: en el Norte bajo la influencia la subregión leonesa y en el Sur de la subregión salmantina, constatación basada en criterios funcionales.

En segundo lugar, los espacios resultantes de la superposición y acople con territorios vecinos, caso de la subregión Oeste de la región, formada por las provincias de León-Zamora-Salamanca, que atendiendo a rasgos geohistóricos y culturales recibe también la denominación de País leonés, restando aún ciertos espacios periféricos de la región fácilmente demarcados, como el tramo correspondiente de la Raya Ibérica, espacio común de vecindad sentido por sus habitantes (los rayanos) y poblaciones (rayanas) en un radio de decenas de kilómetros a ambos lados de la misma, que opera de hecho como laboratorio de políticas de cooperación transfronteriza, o asimismo, la vertiente sur de la Cordillera Cantábrica y otros ámbitos como el Corredor del Duero, que acoge a los municipios ribereños y zonas próximas en una franja imprecisa.

En tercer lugar, la provincia tiene la virtud de poseer una delimitación de tipo administrativo, que, como tal entidad territorial, arranca en España en 1833, pero tiene el defecto pernicioso de no ser un ámbito geográfico per se, pues su trazado fue en su origen para delimitar los territorios de la administración periférica del Reino, y, en ocasiones, alberga aún enclaves territoriales de unas provincias en otras (y de unos municipios en otros), como vestigios del Antiguo 
Régimen. Sin embargo, es evidente que la provincia "ha calado" en toda su extensión y hoy tiene una "carga" a la hora de acometer operaciones de reforma de la organización territorial del Estado, como se desprende de la presencia de siete Comunidades Autónomas uniprovinciales y de la identidad provincial por parte de los ciudadanos, sea en las Comunidades más extensas por razón de distancias (Castilla y León, Castilla-La Mancha, Andalucía), sea en las biprovinciales por motivo de competitividad (Extremadura, Canarias) o, sea sobre todo, en las forales por causa de los derechos históricos (País Vasco). En suma, la provincia se ha consolidado con el tiempo y ha tenido un impacto indudable en la estructura territorial española del que es difícil desprenderse, a pesar de la introducción de nuevas escalas intermedias, como se advierte recientemente con ocasión del proyecto ley de veguerías por parte de la Administración autonómica de Cataluña.

En cuarto lugar, adoptando la escala comarcal, las divisiones realizadas hasta ahora en Castilla y León son de origen meramente administrativo y puestas más bien al servicio de ciertos Ministerios de la Administración Central o de las Consejerías de la Administración Autonómica, salvo en el caso específico de la creación de la comarca de El Bierzo (Ley de 1991). A pesar de ello, no se ha llegado a establecer aquí una demarcación de agrupaciones territoriales intermedias y supramunicipales (caso de las áreas urbanas), como así tendría que haber sido, aplicando una lógica racional territorial. Resta, eso sí, una demarcación tradicional, revisada en sucesivas ocasiones, como es la del partido judicial, que ha dejado su poso e influencia en la percepción del territorio por parte de los ciudadanos en la sociedad rural ("soy del partido de..."), a falta de otro tipo de acepción para posicionarse cada cual entre "su" municipio y "su" provincia.

En este contexto, la comarca tradicional ha periclitado en gran medida, aunque subyace en el imaginario popular. Si tuviésemos que destacar alguna comarcalización señera, sería la del Ministerio de Agricultura, en la que se establece un diseño de comarcas agrarias agrupando municipios con criterios bien equilibrados a efectos de ordenación de sus oficinas y programas, tratándose de una división que ha tenido impacto en documentos posteriores de comarcalización, tal como el diseño de espacios comarcales de actuación (EYSER-Junta de Castilla y León, Estudio y propuesta de comarcalización, 1985), que ha servido de base para actuaciones regionales de descentralización y desconcentración en los ámbitos educativo o sanitario, zonas de acción social, en la demarcación de El Bierzo o en la más reciente de áreas funcionales, asociada al proyecto de Directrices de Ordenación del Territorio (TALLER DE IDEAS, 1996), que, en cierto modo, tal como discurre su tramitación, pasa a ser una geoidea más técnicopolítica de la Administración. 
A la vista del esquema de organización territorial aplicado al caso de Castilla y León resaltan también ciertos aspectos de relevancia geopolítica, tales como la débil conciencia y percepción regional de sus habitantes, al lado de un dominante provincialismo, producto de una forma de ejercer el poder y vista la provincia más que como unidad administrativa como marco de referencia social y coordenadas espaciales de sus ciudadanos, de manera que las interrelaciones son más bien de coexistencia y de recelo hacia la propia autonomía y, de paso, el centralismo vallisoletano, apareciendo además dos fuerzas identitarias enfrentadas, el castellanismo y el leonesismo, como se desprende de diversas encuestas sociológicas a lo largo de estos años (HERNÁNDEZ SÁNCHEZ, 2007: 2028). El gobierno del territorio tiene además, entre otros problemas, una excesiva compartimentación municipal, si nos atenemos a que su cifra supone una cuarta parte del número de municipios españoles, la presencia de enclaves administrativos que anteriores al régimen provincial y municipal y que rompen el principio de continuidad, y una carencia de entidades supramunicipales y ámbitos de escala intermedia (comarcas) para coordinar las esferas de influencia de ciudades y la mayor articulación del territorio regional, como se viene advirtiendo en repetidas ocasiones en los estudios de geógrafos y en las mismas Directrices de Ordenación Territorial de Castilla y León (TALLER DE IDEAS, 1996, 2000).

Advertir también que, en territorio tan extenso y relativamente poco poblado se suman rasgos diferenciadores geográficos como la existencia de amplias distancias entre sus ciudades y la de áreas periféricas muy compartimentadas por la orografía, la vecindad de la metrópoli de Madrid que ejerce de nodo y centro radial de buena parte de las comunicaciones que atraviesan la región, o el hecho de que la región sea plataforma de intercomunicación o interfaz entre nueve regiones españolas y dos portuguesas.

Conviene tener en cuenta también la situación de las piezas de articulación que inciden en las políticas de ordenación territorial, tales como las siguientes:

$1^{\circ}$ ) Presencia de estructuras geométricas de interrelación de nodos, del tipo de corredores lineales (en particular el del Duero, entre Zamora y Aranda, con tendencia a prolongarse en la dirección de Soria), corredores diagonales (así el que atraviesa todo el territorio entre Fuentes de Oñoro, en la misma frontera con Portugal, y Miranda, ciudad-puerta en relación al valle del Ebro y al País Vasco), triángulos (de diferente dimensión, como los de León-Astorga-Benavente, Burgos-Valladolid-Aranda y el más amplio de León-Burgos-Salamanca y que interrelaciona a las siete mayores ciudades de la región) o el pentágono (que encierra el territorio central de la región prolongando los triángulos centrales hacia el sur y este). 
$\left.2^{\circ}\right)$ Puertas con el exterior de la región, como las ciudades de Miranda de Ebro, Segovia, Soria, Ponferrada, Ciudad Rodrigo, Béjar, más alguna villanodo de comunicaciones por carretera como Aguilar de Campoo y pequeños nodos con esta vocación como Medinaceli o Puebla de Sanabria.

$\left.3^{\circ}\right)$ Asentamientos o nodos centrales de articulación en la región, en sus distintos niveles jerárquicos, junto a centros exteriores a la región con la que conectan y ejercen mayor o menor influencia (Madrid, Bilbao, Zaragoza, preferentemente, además de otras ciudades como Santander, Oviedo, Talavera de la Reina, Vitoria o Logroño).

En definitiva, el marco territorial y los rasgos regionales convierten a Castilla y León en una pieza del territorio en red del Suroeste de Europa y en particular de los Cuadrantes Noroeste y Norte ibérico. Asimismo, la ejecución de grandes infraestructuras plantea un refuerzo e incremento de la interacción entre las diferentes regiones y ciudades, haciéndose muy necesaria una política de ordenación y actuación territorial desde una visión ibérica, sirviendo la región de conexión con nodos clave en la red logística y de transportes de carretera (Miranda de Ebro, Benavente y Tordesillas) o de ferrocarril (Miranda de Ebro, Palencia-Venta de Baños, León, Medina del Campo), a la vez que juega un papel de encrucijada y de traspaís para las regiones atlánticas españolas y portuguesas del "Arco Atlántico".

\section{DIAGNOSIS SUCINTA Y PROSPECTIVA DE ORDENACIÓN TERRITORIAL}

Desde una visión socioeconómica, el panorama de Castilla y León tal como se desprende de ciertas variables estructurales, comparado con el de España, según la consulta de valores reflejados en alguna fuente de crédito (CES, 2009), presenta los siguientes rasgos generales:

A) En primer término, un territorio con una superficie de $94.225 \mathrm{~km}^{2}(18,6 \%$ de España), mientras que la población a 1 de enero de 2010 era de 2.555 .715 habitantes $(5,4 \%$ del total) y la población inmigrante extranjera residente era de 167.597 personas (apenas un 2,9\% de la establecida en el país), contando con una densidad poblacional de $27 \mathrm{~h} / \mathrm{km}^{2}(29,4 \%$ de la tasa correspondiente española), con una relativa concentración en tasas mayores en áreas urbanas y ciertas áreas rurales -FIGURA 6-, mientras que la tasa de natalidad es inferior a la española y las de mortalidad y envejecimiento son superiores, delatando el estancamiento de la población regional con tendencia a un cierto retroceso, que no salva el saldo migratorio positivo de los últimos años.

Es reconocible la ausencia de presión demográfica sobre el territorio de esta región, caracterizada, al contrario, por ser un territorio de extensos vacíos y dé- 
bil densidad de población. Sin embargo, lo negativo es sobre todo que no haya apenas niños y jóvenes en muchos de los pueblos, que la mortalidad sea un año tras otro superior a la natalidad en la mayor parte de los núcleos rurales y semiurbanos, que la crisis demográfica reste capacidad de recursos humanos para el desarrollo, dinámica y atracción de las ciudades, o que haya entrado menos inmigración extranjera en el último decenio en la región con relación a la llegada a España... y no tanto que haya bajas densidades demográficas, ajustadas como están a los potenciales y limitaciones de las explotaciones agrarias y a las nuevas relaciones campo-ciudad, siendo esta la que marca la dirección de concentración demográfica principalmente.

Figura 6. Densidad de población, 2008

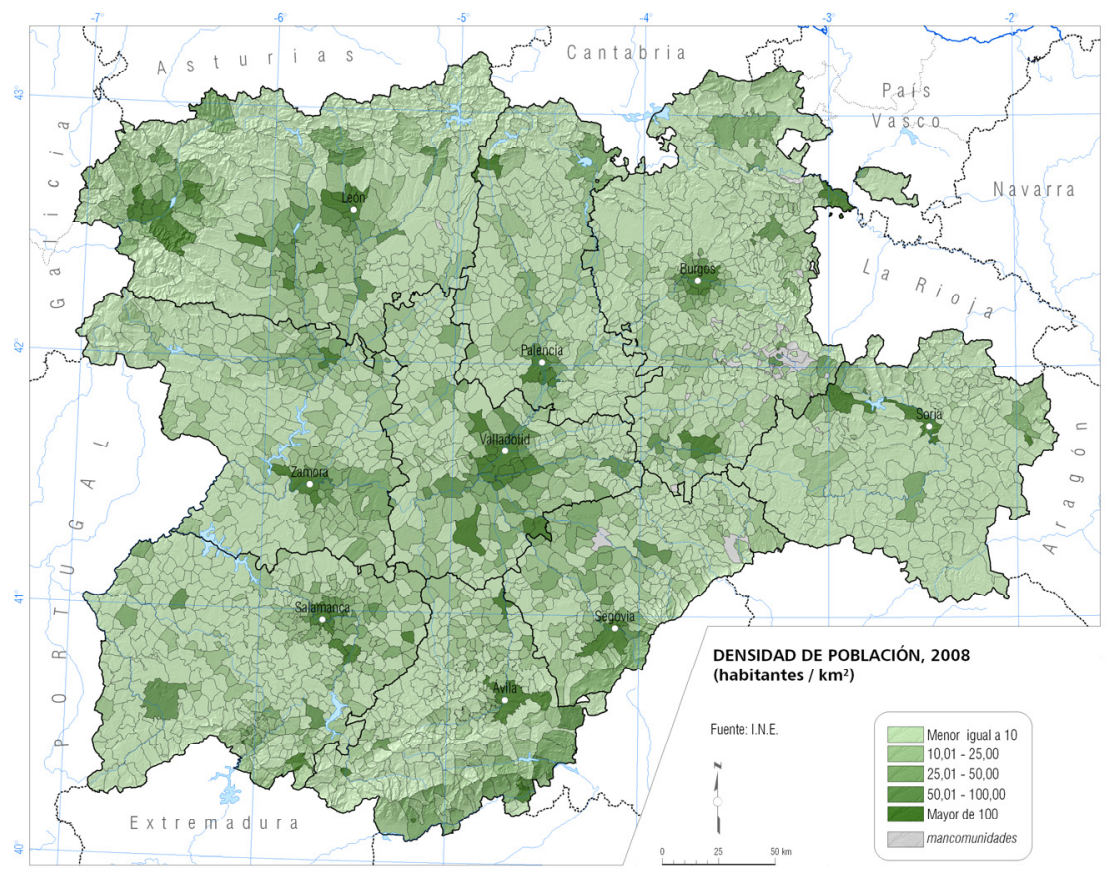

De ahí que el proceso de despoblamiento rural y de concentración urbana se haya traducido en un desajuste espacial salpicado por vacíos demográficos, comprometiendo a medio y largo plazo el ritmo de crecimiento demográfico por la falta de vitalidad vegetativa, si bien en algunos espacios asistimos a un cierto "renacimiento" en cuanto a población (municipios de Segovia próximos a Madrid) y diversificación de actividades económicas (El Bierzo y Ribera del Duero). Mientras que el medio urbano aglutina a la mayor parte de la población regional y no se limita a los enclaves urbanos sino que se ensancha a través de un 
periurbano de concentración demográfica en forma de aureolas periurbanas en proceso de dispersión, rompiéndose así el viejo esquema de las relaciones campo-ciudad. Otros aspectos a tener en cuenta son la localización de los asentamientos a lo largo de grandes ejes de transporte o en ciertas áreas debido al fenómeno de segunda residencia, ocupada esencialmente por población residente en metrópolis de fuera de la región y en las ciudades regionales.

Derivado de todo ello, se puede entender esta distribución espacial de la población en un doble sentido, bien como problema, esencialmente en los núcleos rurales, en cuanto a la naturaleza de la relación que se establece entre la densidad y número de asentamientos y su tamaño, los rasgos de avanzado proceso de envejecimiento, falta de vitalidad por la presencia de generaciones huecas en la mayoría de núcleos, la estacionalidad del medio rural debido a la ocupación vacacional por residentes de la ciudad, etc., o bien como oportunidad y potencialidad, si se atiende el territorio como recurso a valorar, donde las potencialidades vienen dadas por la disponibilidad de grandes espacios de notable calidad ambiental y paisajística, la escasez y localidad de los conflictos de usos y la posibilidad de insertar en este medio actividades económicas.

B) En segundo término, el PIB regional representaba en 2008 el 5,3\% del español, situado muy cercano al promedio de las regiones y, como se puede observar, afín a la contribución de los recursos de población de la región. Mientras que se establece un valor de la participación sectorial en la productividad y empleo no tan diferente del promedio español, si bien con desajustes en cuanto a una mayor participación de Castilla y León en la Agricultura, y de los Servicios, a la inversa, con una mayor participación en España. Asimismo se aprecia cada vez más la aportación al crecimiento regional de Castilla y León por parte de los servicios y las industrias del automóvil y de la alimentación.

Tabla 1. Participación y aportaciones al crecimiento, en porcentajes, 2008

\begin{tabular}{lrr}
\hline \multicolumn{1}{c}{ Sector } & Castilla y León & España \\
\hline Agricultura & 7,9 & 3,2 \\
Industria & 20,4 & 17,9 \\
Construcción & 9,9 & 9,2 \\
Servicios & 61,8 & 69,7 \\
\hline
\end{tabular}

FUENTE: Contabilidad Nacional Trimestral y grupo Hispalink-Castilla y León.

Domina en esta región, pues, el recurso territorial sobre el demográfico, caracterizado más por los territorios de la despoblación y el envejecimiento (LÓPEZ TRIGAL, 2009) que por los de la concentración urbana y se mantiene un mayor grado de ruralidad y peso del sector primario, en especial en el occidente de la región (las tres provincias de León, Zamora y Salamanca) que en el ámbito nacional. Asimismo, se puede observar en el último decenio una convergen- 
cia de la región en el valor de Renta per capita con el índice medio español, como también en el modelo de crecimiento económico en cada caso.

A partir de los datos básicos anteriores y, desde una visión integrada, los rasgos y problemas de la región que afectan a la articulación territorial se pueden situar a partir de dos procesos paralelos:

$1^{\circ}$ ) Un avance de los fenómenos de despoblación y envejecimiento que desemboca en un estancamiento con tendencia al declive demográfico y en la crisis agónica agraria, debida a las dificultades de mejora de las explotaciones, en buena medida subsidiadas y con insuficiente competitividad, presentándose de este modo amplios territorios vacíos en el interior de la región y sobre todo en las áreas periféricas.

$2^{\circ}$ ) Un proceso de concentración demográfica y de actividades que aboca al desequilibrio territorial de las actividades industriales, concentradas relativamente en el eje Valladolid-Burgos, como se observa a través de la localización en el mismo de las doce primeras empresas de la región, pertenecientes al sector de la automoción (Renault, Michelin, Iveco, Firestone, Antolín, TRW, Nissan) y de la alimentación (Unigro, Campofrío y Pascual), que polarizan a su vez el comercio exterior.

De este modo, se podría caracterizar la región como relativamente desequilibrada, aunque en ningún caso "desarticulada territorialmente", en cuanto que coincide en gran medida con el ámbito de una región física como es la cuenca española del Duero y la Submeseta Norte, la red de ciudades se encuentra bien jerarquizada y de tipo policéntrico lo que anima a la competitividad urbana, con una centralidad cada vez más destacada por parte de la capital política regional, Valladolid, pero repartiendo juego con las tres capitales subregionales, Burgos, León y Salamanca, localidades centrales de un mismo nivel jerárquico superior, mientras que restan en un nivel jerárquico intermedio las cuatro capitales provinciales restantes más Ponferrada y a distancia una decena de pequeñas ciudades entre 10.000 y 30.000 habitantes, de un nivel básico urbano y de un número insuficiente y dinámica reducida en general.

De otro lado, siguiendo un análisis DAFO aplicado, podemos advertir un diagnóstico socioeconómico y territorial en el que la región presenta como potencialidades: a) un marcado policentrismo urbano unido a cierta dimensión de los propios recursos territoriales, naturales y culturales, b) una relativa posición y renta geográfica de proximidad respecto a los diferentes polos metropolitanos ibéricos y en especial a la región urbana de Madrid, c) unas fuentes energéticas diversificadas así como por parte de la producción agraria, en particular las ganadera y vitícola, d) la dinámica favorable que aportan a la economía regional 
las industrias de automoción y de la alimentación, e) el relativo desarrollo de la red de transportes terrestres y plataformas logísticas, f) las universidades y redes de servicios y g) una amplia oferta de turismo interior y cultural, de primer orden en España.

Mientras que, por su lado, como amenazas nos topamos con a) el declive del saldo natural demográfico, b) el envejecimiento de la población, c) la atracción y absorción de fuerzas y dinámicas desde el centro madrileño, d) la permanente crisis de muchos de los subsectores agrarios y el mundo rural, e) las dificultades de creación de empleo y dinamización industrial, entre otras, además de f) una localización territorial en buena parte periférica y alejada de los centros más dinámicos, particularmente los situados en el oeste de la región y en la orla montañosa.

Ante esta diagnosis, se opera con instrumentos de animación de los recursos y de los sectores económicos y se han seguido en los últimos tiempos diversas políticas públicas por parte de las diferentes Administraciones y en especial las referentes a la política de cohesión europea, como es el caso de la cooperación transfronteriza, un nuevo ámbito de dinamización e integración territorial entre España y Portugal con el que se pretende superar su situación periférica secular desde la mejora de la accesibilidad y la apertura de nuevos escenarios de desarrollo y de ordenación y planificación conjunta con programas y actuaciones en las dos últimas décadas que abarcan todo tipo de objetivos en cuanto a animación y consolidación del desarrollo endógeno del área (LÓPEZ TRIGAL, 2005). Asimismo, se asiste a diferentes planes especiales de actuación en áreas de reconversión minera, áreas periféricas de la región, etc. por parte de la Administración de Castilla y León.

Otro supuesto diferente en este contexto es la promoción de los sectores emergentes y con buenas perspectivas para el futuro de la economía regional, como son básicamente "los relativos a actividades de logística, agroalimentarias y del conocimiento, por lo que el mayor desarrollo de estas actividades puede suponer un claro factor de especialización de nuestra economía en actividades de alto valor añadido y contribuir a diversificar nuestra estructura productiva" (CES, 2009, tomo II: 94). En esta línea, es notable el esfuerzo desarrollado por las instituciones públicas y en especial las ciudades en asumir programas y políticas de desarrollo asociados a la dinamización e innovación, con apoyo en actuaciones muy diferentes, como son la creación de parques tecnológicos (Valladolid-Boecillo, Burgos, León), centros de congresos y culturales (Salamanca, Valladolid, Burgos, León...), aeropuertos (Valladolid, León, Salamanca, Burgos), enclaves logísticos (red de Centros CYLOG en Palencia-Área Central, Valladolid, León, Ponferrada, Zamora, Benavente, Salamanca, Ávila, Arévalo, Segovia, Aranda de Duero, Burgos, Miranda de Ebro, Soria), o la integración ur- 
bana de estaciones de alta velocidad ferroviaria (Burgos, Palencia, Valladolid, León...) y todo ello asociado a la promoción del desarrollo regional y urbano.

Un ejemplo singular, de línea programática y estratégica regional institucionalizada, es el 'Plan de Internacionalización de Castilla y León', 2008-2011, en la actualidad al final de su segunda fase. Se trata de una plataforma desarrollada por la Junta de Castilla y León, el Instituto de Comercio Exterior y las Cámaras de Comercio e Industria para impulsar la expansión de "empresas tractoras" de la región y de excelencia innovadora $(\mathrm{I}+\mathrm{D}+\mathrm{i})$, promoviendo mayor diversificación y alianzas empresariales, impulsando parques empresariales, captando inversiones de empresas multinacionales y movilizando sectores estratégicos del transporte, la biotecnología, las energías renovables, entre otros. Su objetivo se concreta ahora en "duplicar la cifra de exportación de las empresas regionales no ligadas al sector de la automoción", ya que este aún retiene buena parte del porcentaje de las exportaciones regionales (un $69,6 \%$ en 2003 y reducido a un $58,2 \%$ en 2007).

Se aspira, en suma, a la adaptación a una nueva economía a partir de "islas urbanas de innovación", "hub de centros tecnológicos-científicos y de infraestructuras en red", aunque este diseño estratégico aparezca en ocasiones envuelto en objetivos de marketing urbano (caso del Plan Regional del Valle del Duero que incluye la iniciativa de ejecución de cinco edificios singulares o "cúpulas del Duero", promoción de nuevas infraestructuras polivalentes, revestidas a la vez de lo ecológico y la promoción inmobiliaria en el caso de Zamora), que tienen en parte el riesgo de caer en una política de fachadismo y de marca de ciudad y región, lo que no es un asunto secundario.

En fin, el papel que corresponde a la posición de Castilla y León en el marco suprarregional y de espacios-proyecto ibéricos está, como advertíamos anteriormente, en ejercer y servir de rótula o espacio-bisagra entre la Fachada Atlántica y las Regiones Interiores capitaneadas por Madrid, a través de una apuesta por la intermodalidad y la prosecución de nuevas infraestructuras de comunicación (autovías y autopistas de peaje, trazado ferroviario de alta velocidad), y se aspira a hacer frente a los efectos de la radialidad con el reforzamiento de los ejes del Subcantábrico, del Duero o de la Vía de la Plata, tanto en el Plan nacional (PEIT) como en el Plan regional de infraestructuras.

Mientras tanto, por parte de los agentes regionales, públicos como privados, se fomenta la integración con las regiones del Noroeste y más recientemente del Oeste en su posicionamiento en los grandes corredores terrestres europeos e ibéricos, así como en situarse ventajosamente en los hinterland portuarios y metropolitanos, mientras que, curiosamente, apenas se ha tratado, hasta ahora, de 
cooperar con Madrid o el País Vasco sino que más parece que se trata de estar de espaldas a los centros más dinámicos y competitivos de nuestra vecindad.

Por otro lado, la región ha de potenciar su sistema urbano policéntrico, tanto en los primeros centros (la capitalidad regional y las capitales subregionales), cuanto en los centros intermedios y pequeñas ciudades, junto a las cabeceras y polos comarcales y locales que completan la red de lugares centrales, así como hacer frente al desafío de la reconfiguración y reordenación territorial y administrativa pendiente de diseño y demarcación de comarcas y de áreas urbanas, labor esta esencialmente de signo geográfico como político pendiente en los últimos tiempos.

En esa línea, se deberían adoptar por la Administración autonómica medidas de calado político en el marco de las Directrices Territoriales: 1) Reajuste de la demarcación territorial municipal y a la vez impulso de la descentralización en entidades locales menores, reduciendo radicalmente el número de municipios con el fin de que sean garantes de la prestación de servicios básicos para una población mínima de 2.000 a 10.000 habitantes, además de la supresión de los enclaves de ámbito inframunicipal siguiendo el principio de continuidad territorial. 2) Diseño de la escala intermedia entre municipio y provincia a partir de la puesta a punto del municipio-comarca y espacios funcionales, comenzando por el ámbito de las áreas urbanas para la coordinación necesaria de la gestión supramunicipal, así como la selección de las respectivas cabeceras y núcleos de interés territorial que acojan la provisión y distribución en su territorio de los servicios públicos. 3) El equilibrio de la ocupación del territorio a partir del impulso del policentrismo en las diferentes escalas del sistema urbano y territorial, es decir, de tipo regional, provincial y también en la esfera comarcal.

Estos retos, programas y revisiones críticas de desigual relevancia se presentan en el marco de la región castellano-leonesa, que necesita ajustar aún más su nivel de articulación territorial, urbana y socioeconómica, tal como venimos advirtiendo los geógrafos de la región, acaso también por ser nuestra primera preocupación profesional en los últimos tiempos, en una línea de acercamiento de la Geografía y la Ordenación del Territorio en el ámbito de nuestro campo de formación y acción. Pero, mantener y desarrollar el estudio y análisis geográfico regional de la investigación en sus diferentes escalas, desde la macrorregional a la subregional junto a los estudios de casos locales, no ha de mermar nuestro interés por situar el análisis regional en una línea aplicada y estratégica de propuestas y proyecciones. 


\section{BIBLIOGRAFÍA Y DOCUMENTACIÓN}

AAVV (1982): El espacio geográfico de Castilla la Vieja y León, Burgos, Consejo General de Castilla y León.

AAVV (1987): Castilla y León, Madrid, Junta de Castilla y León.

AAVV (1988): Análisis del medio físico, Valladolid, Junta de Castilla y León, 9 vols.

AAVV (1998): La Economía de Castilla y León ante el siglo XXI, Valladolid, Junta de Castilla y León.

BANCO DE BILBAO (1971): Panorama económico castellano-leonés, Bilbao, Editorial Eléxpuru Hermanos.

CABERO, V. (1982): El espacio geográfico castellano-leonés, Valladolid, Ámbito.

CABO Alonso, A. (1975): «Castilla la Vieja y León. Introducción geográfica», en AAVV, Tierras de España, Barcelona, Noguer, págs. 11-53.

Cabo Alonso, A. y Manero Miguel, F., dirs. (1987-1992): Geografia de Castilla y León, Valladolid, Ámbito, 10 volúmenes.

Cabo Alonso, A. y Manero Miguel, F. (1990): «Castilla y León», en AAVV, Geografía Regional de España, Barcelona, Planeta, vol. 6, 255-591.

CARASA, P., coord., (2003): La Memoria histórica de Castilla y León, Salamanca, Junta de Castilla y León.

CARrasco MuÑoz DE Vera, C. (1978): La alternativa de Castilla y León. Datos para una verdadera Autonomía, Madrid, Ediciones de la Torre.

CES (L, LÓPEZ TRIGAL, dir.) (1998): La población en el desarrollo de Castilla y León, Consejo Económico y Social de Castilla y León, Valladolid.

CES (L. LóPEZ TRIGAL, dir.) (2003) La Ordenación del Territorio en Castilla y León, Consejo Económico y Social de Castilla y León, Valladolid.

CES (2009): Situación económica y social de Castilla y León en 2008, Valladolid, Consejo Económico y Social de Castilla y León, 2 tomos.

EYSER (1981): Estudios de reconocimiento territorial de la región castellano-leonesa, Madrid (policopiado).

EYSER (1984-1985): Estudio y propuesta de comarcalización de Castilla y León, Valladolid, Junta de Castilla y León, Consejería de Presidencia (varios volúmenes policopiados).

GARCÍA FERNÁNDEZ, J. (1968): «Submeseta Septentrional. Castilla la Vieja y León», en M. DE TERÁn y L. SOLÉ SABARÍs (dirs.), Geografía Regional de España, Barcelona, Ariel, 100-154.

GARCÍA FERnÁNDEZ, J. (1981): Desarrollo y atonía en Castilla, Barcelona, Ariel.

GARCÍA FERNÁNDEZ, J. (1985): Castilla (entre la percepción del espacio y la tradición erudita), Madrid, Espasa Calpe.

HERNÁNDEZ SÁNCHEZ, A. (2007): Identidad, autonomía y conciencia regional en Castilla y León, Valladolid, Universidad de Valladolid.

LÓPEZ TRIGAL, L. (1980): «Los inicios de la publicación geográfica sobre León», Estudios Humanísticos, ${ }^{\circ}$ 2, 125-129.

LÓPEZ Trigal, L. (1987): Geografía humana de Castilla y León, Barcelona, Oikos-tau. LóPez Trigal, L. (1994): «Castilla y León», en C. CARRERAS Verdaguer y A. GARCÍA BALlesteros (dirs.), Geografía de España, Barcelona, Instituto Gallach, vol. 8, págs. 1.446-1.550. 
LÓPEZ TRIGAL, L., (2005): «La Raya Ibérica como espacio de atracción y cooperación», Papeles de Economía Española, Economía de las Comunidades Autónomas, nº 21, 3- 13.

LÓPEZ TRIGAL, L. (2009): «Despoblación y reconfiguración territorial en España», en L. López Trigal, A. Abellán García y D. Godenau, coords., Envejecimiento, despoblación y territorio, León, Universidad de León, págs. 529-546.

MANERO, F. (1983): La industria en Castilla y León (dinámica, caracteres, impacto), Valladolid, Ámbito.

MANERo Miguel, F. (1987): «Castilla y León», en M. DE TERÁN, L. SOLÉ SABARís y J. VILÁ VALENTí, dirs., Geografía Regional de España, Barcelona, Ariel, $5^{\text {a }}$ edición, 167-244.

MOLINERO, F. (1982): El regadio, ¿una alternativa a la agricultura castellanoleonesa?, Valladolid, Ámbito.

PASTOR ANTOLÍn, L. J. (1986): La red de transporte en Castilla y León, Valladolid, Ámbito.

Revista de Economía y Finanzas de Castilla y León (2004): «Territorio y planificación, una aproximación a Castilla y León», $n^{\circ} 9$.

Sorel, A. (1975): Castilla como agonía, Madrid, Ediciones del Centro.

TALLER DE IDEAS (1996): Directrices de Ordenación Territorial. Castilla y León, Hipótesis de modelo territorial, Madrid, Junta de Castilla y León.

TAller De IdEAS (2000): Directrices de Ordenación del Territorio. Castilla y León, Salamanca, Junta de Castilla y León.

TERÁN, M. de (1958): «La meseta septentrional», en M. DE TERÁN y OTROS, Geografía de España y Portugal, Barcelona, Montaner y Simón, vol. IV, 93-162. 